\title{
Stage III Pleural Malignant Mesothelioma AJCC v8
}

National Cancer Institute

\section{Source}

National Cancer Institute. Stage III Pleural Malignant Mesothelioma A/CC v8. NCI

Thesaurus. Code C136403.

Stage III includes: IIIA: (T3, N1, M0); IIIB: (T1-3, N2, M0); (T4, Any N, M0). T1: Tumor limited to the ipsilateral parietal with or without involvement of visceral pleura, mediastinal pleura, and diaphragmatic pleura. T2: Tumor involving each of the ipsilateral pleural surfaces (parietal, mediastinal, diaphragmatic, and visceral pleura) with at least one of the following features: involvement of diaphragmatic muscle and/or extension of tumor from visceral pleura into the underlying pulmonary parenchyma. T3: Tumor is locally advanced but potentially resectable. The tumor involves all the ipsilateral pleural surfaces (parietal, mediastinal, diaphragmatic, and visceral pleura) with at least one of the following features: involvement of the endothoracic fascia, extension into the mediastinal fat, solitary, completely resectable focus of tumor extending into the soft tissues of the chest wall, and/or nontransmural involvement of the pericardium. T4: Tumor is locally advanced and technically unresectable. The tumor involves all the ipsilateral pleural surfaces (parietal, mediastinal, diaphragmatic, and visceral pleura) with at least one of the following features: diffuse extension or multifocal masses of tumor in the chest wall, with or without associated rib destruction, direct transdiaphragmatic extension of tumor to the peritoneum, direct extension of tumor to the contralateral pleura, direct extension of tumor to mediastinal organs, direct extension of tumor into the spine, and/or tumor extending through to the internal surface of the pericardium with or without a pericardial effusion; or tumor involving the myocardium. N1: Metastases in the ipsilateral bronchopulmonary, hilar, or mediastinal (including the internal mammary, peridiaphragmatic, pericardial fat pad, or intercostal) lymph nodes. N2: Metastases in the contralateral mediastinal, ipsilateral, or contralateral supraclavicular lymph nodes. M0: No distant metastasis. (AJCC 8th ed.) 